\title{
Estado nutricional e produtividade da seringueira em solo com calcário aplicado superficialmente
}

\author{
Cassiano Garcia Roque ${ }^{(1)}$, Renato de Mello Prado ${ }^{(1)}$, William Natale ${ }^{(1)}$, Amauri Nelson Beutler ${ }^{(1)}$ e José Frederico Centurion ${ }^{(1)}$
}

(1)Universidade Estadual Paulista, Fac. de Ciências Agrárias e Veterinárias, Dep. de Solos e Adubos, Via de Acesso Prof. Paulo Donato Castellane, s/nº, CEP 14870-000 Jaboticabal, SP. E-mail: cgroque@fcav.unesp.br, rmprado@fcav.unesp.br

\begin{abstract}
Resumo - A aplicação de calcário superficial na seringueira em produção pode melhorar a fertilidade do solo e a produção de borracha seca. O objetivo deste trabalho foi avaliar o efeito da aplicação superficial de calcário no solo na produtividade de borracha seca. $\mathrm{O}$ experimento foi instalado em seringal em produção (15 anos), num Argissolo Vermelho-Amarelo com saturação por bases (V) de 39\%. Os tratamentos constituíram-se das seguintes doses de calcário calcinado: zero, metade da dose recomendada, uma vez a dose recomendada e duas vezes a dose recomendada, correspondendo a 0, 0,21, 0,42 e 0,84 $\mathrm{tha}^{-1}$, respectivamente. As doses foram calculadas considerando a saturação por bases recomendada para a cultura no Estado de São Paulo, igual a 50\%. A aplicação de calcário neutralizou a acidez do solo e aumentou a saturação por bases até a camada de $0,20 \mathrm{~m}$, e aumentou a produtividade da borracha seca. A produtividade máxima esteve associada à saturação por bases de $57 \%$ e teor foliar de Ca de $8 \mathrm{~g} \mathrm{~kg}^{-1}$.
\end{abstract}

Termos para indexação: Hevea brasiliensis, calagem, pH, nutrição, borracha seca.

\section{Nutritional status and productivity of rubber tree on soil with surface applied lime}

\begin{abstract}
The surface lime application in rubber tree (Hevea brasiliensis) during the productive phase can improve the fertility of the soil and the dry rubber yield. The objective of this work was to evaluate the effect of soil surface lime application on the dry rubber yield. The experiment was installed in mature rubber tree (15 years), in a Typic Kandiuldult with saturation for bases (V) of 39\%. The treatments were constituted by doses of calcined liming: zero, half of the recommended dose, a time the recommended dose and two times the recommended dose, corresponding to $0,0.21,0.42$ and $0.84 \mathrm{t} \mathrm{ha}^{-1}$, respectively. The doses were calculated considering the base saturation for the culture (V=50\%) in São Paulo State. Lime application neutralized the soil acidity and increased the base saturation until the layer of $0.20 \mathrm{~m}$ and increased dry rubber yield. The maximum yield was associated with base saturation of $57 \%$ and with foliar Ca content of $8 \mathrm{~g} \mathrm{~kg}^{-1}$.
\end{abstract}

Index terms: Hevea brasiliensis, liming, soil $\mathrm{pH}$, nutrition, dry rubber.

\section{Introdução}

O cultivo da seringueira (Hevea brasiliensis Muell Arg.) planta originária da Região Amazônica, pode alcançar alta produção de borracha, quando empregados clones melhorados e tratos culturais adequados. Os seringais implantados no planalto Ocidental do Estado de São Paulo vêm apresentando bons resultados de crescimento e produção. No entanto, fatores edáficos exigem cuidados nas áreas de adubação e nutrição da cultura, de forma a não limitar o crescimento e a produção da seringueira (Murbach et al., 1999).

No controle da acidez dos solos tropicais, a calagem mostra-se uma técnica com baixa relação custo/benefício, em diversas culturas, já que a aplicação de calcário reduz a acidez do solo, diminui a concentração de $\mathrm{Al}$ e
Mn do solo e aumenta a disponibilidade de nutrientes e a atividade da microbiota do solo. Estes efeitos combinados resultam na adequada nutrição da planta, maior crescimento radicular e da parte aérea e, conseqüentemente, maior produção (Prado, 2003). Schwengber (1993), em seringueira (clone IAN-873) cultivada em Latossolo Vermelho-Escuro, argiloso, constatou que o nível crítico de Ca usando o crescimento da raiz foi de $1,5 \mathrm{mmol}_{\mathrm{c}} \mathrm{dm}^{-3}$. Assim, existem indicações do uso de calcário dolomítico para a seringueira apenas como fonte de Ca e Mg (Shorrocks, 1979).

Domingues (1994) constatou que 20 seringais localizados em regiões do Planalto do Estado de São Paulo apresentaram acidez elevada, baixos teores de P, K, Ca e $\mathrm{Mg}$ e baixa saturação por bases. Verificou também que os seringais situados em solos com saturação por 
bases superior a $50 \%$ apresentaram um maior incremento anual no perímetro do tronco. Bataglia et al. (1988) notaram, em seringais paulistas, que as glebas com baixa produtividade $\left(<1,0 \mathrm{t} \mathrm{ha}^{-1}\right)$ estavam associadas a solos com acidez mais elevada $(\mathrm{V}=27 \%)$. Apesar de trabalhos sobre a reação da seringueira à calagem serem ainda insuficientes, a calagem é recomendada para elevar V a 50\% no Estado de São Paulo (Cardoso, 1992).

Os poucos resultados experimentais revelam resposta positiva da seringueira à aplicação de calcário. Assim, Virgens Filho et al. (2001) avaliaram seringais do clone RRIM 600 no planalto paulista submetidos à aplicação de calcário superficial, em um Latossolo Amarelo álico, textura média $(\mathrm{V}=14 \%)$, objetivando elevar $\mathrm{V}$ a 50\%. Estes autores obtiveram maior produtividade de borracha seca apenas quando a calagem foi seguida de adubação, comparada à área apenas com adubação e sem calagem. Já Reis et al. (1984) não obtiveram resposta da seringueira à calagem em experimento realizado no campo no Sul da Bahia. Verificaram também que a aplicação conjunta de NPK e calcário (ausência e presença) não permitiu obter a curva de calibração e identificar o valor ideal de $\mathrm{V}$ para essa cultura.

Em casa de vegetação, houve resposta positiva da aplicação de Ca na massa de matéria seca da seringueira. Entretanto, doses acima de $67 \mathrm{mg} \mathrm{L}^{-1} \mathrm{de} \mathrm{Ca} \mathrm{cau-}$ saram redução no crescimento da planta, embora não tenham afetado os teores de N, P e K na planta (Viegas et al., 2001). Efeitos negativos no desenvolvimento e na nutrição foram constatados com aumento das doses de alumínio (>15 mg L $\mathrm{m}^{-1}$ ) (Bueno, 1987). Em viveiro, Pereira \& Pereira (1987) não observaram resposta da seringueira à aplicação de calcário no solo com pH 4,2 e $5 \mathrm{mmol}_{\mathrm{c}} \mathrm{dm}^{-3}$ de cálcio.

O objetivo deste trabalho foi avaliar o efeito da aplicação superficial de calcário no solo na produtividade de borracha seca da seringueira.

\section{Material e Métodos}

O experimento foi implantado na Fazenda São Roque, situada a $20 \mathrm{~km}$ do Município de Novo Horizonte, SP (21 ${ }^{\circ} 28^{\prime} 2^{\prime \prime} \mathrm{S}, 4^{\circ} 13^{\prime} 17^{\prime \prime} \mathrm{W} ; 453 \mathrm{~m}$ de altitude média), onde o clima é o mesotérmico com inverno seco (Cwa), pelo sistema de Köppen. O solo é um Argissolo Vermelho-Amarelo, que apresentou inicialmente as seguintes características químicas $(0,00-0,20 \mathrm{~m}): \mathrm{pH}, 4,6$; MO, $11 \mathrm{~g} \mathrm{dm}^{-3} ; \mathrm{P}, 6 \mathrm{mg} \mathrm{dm}^{-3} ; \mathrm{K}, 1,5 ; \mathrm{Ca}, 13 ; \mathrm{Mg}, 5 ; \mathrm{H}+\mathrm{Al}$, 30 ; SB, 19,5; T, 49,5 $\mathrm{mmol}_{\mathrm{c}} \mathrm{dm}^{-3} \mathrm{e} \mathrm{V}, 39 \%$. A área ex- perimental possuía um horizonte orgânico de $0,04 \mathrm{~m}$ de espessura, formado por detritos vegetais pouco decompostos.

O seringal (clone RRIM 600) havia sido implantado há 15 anos com espaços de 2,5 m entre plantas e 7,5 m entre linhas. Depois da implantação o seringal não recebeu mais calcário e adubo.

O delineamento utilizado foi o de blocos ao acaso com quatro tratamentos e cinco repetições. A parcela total era constituída de quatro linhas com cinco plantas e a área útil, de seis plantas centrais. Os tratamentos constituíram-se das seguintes doses de calcário calcinado (CaO, $420 \mathrm{~g} \mathrm{~kg}^{-1} ; \mathrm{MgO}, 250 \mathrm{~g} \mathrm{~kg}^{-1} ; \mathrm{PN}, 137 \%$; RE, 96\% e PRNT, 131\%): zero, metade da dose recomendada, uma vez a dose recomendada e duas vezes a dose recomendada, correspondendo a $0,0,21,0,42$ e $0,84 \mathrm{tha}^{-1}$, respectivamente. As doses foram calculadas considerando-se a saturação por bases recomendada para a cultura igual a 50\% (Raij et al., 1996). O calcário foi aplicado manualmente na superfície do solo, sem incorporação, em novembro de 2001.

As amostras de solo foram coletadas em 15 pontos aleatórios na entrelinha da parcela, nas profundidades de $0,00-0,10,0,10-0,20$ e $0,20-0,40 \mathrm{~m}$, com auxílio de um trado tipo holandês, em maio de 2002 e março de 2003, aos seis e 16 meses após a aplicação dos tratamentos, respectivamente.

Para análise química dos macro e micronutrientes, foram coletadas as três folhas recém-maduras sem pecíolo, em ramos sombreados da base do último lançamento (Bataglia et al., 1992), por árvore da parcela útil, em janeiro de 2003, ou seja, 14 meses após a aplicação dos tratamentos. As determinações químicas realizadas no solo e nas folhas foram feitas conforme Raij et al. (2001) e Bataglia et al. (1983), respectivamente.

Avaliou-se a produtividade da borracha seca de janeiro a julho e de outubro a dezembro de 2002 , e de janeiro a julho de 2003. Na avaliação da produção de borracha, foi adotado o sistema $1 / 2 \mathrm{~S} \mathrm{~d} / 56 \mathrm{~d} / 7.10 \mathrm{~m} / \mathrm{y}$ ET $2,5 \% \mathrm{~Pa} 6 / \mathrm{y}$ - sangria em meia-espiral $(1 / 2 \mathrm{~S})$, realizada em intervalos de cinco dias (d/5). Esta atividade foi realizada em seis dias por semana (6d/7). Uma vez por mês, realizou-se a coleta do látex coagulado de uma sangria. Após a coleta, os coágulos foram pesados individualmente, e coletou-se uma amostra de cada um deles para determinação da umidade. A secagem foi realizada em estufa ventilada, a uma temperatura em torno de $65^{\circ} \mathrm{C}$, até atingir peso constante.

Os dados foram submetidos à análise de variância pelo teste $\mathrm{F}$. O efeito das doses de calcário nas carac- 
terísticas químicas do solo, na produtividade da borracha e o efeito da saturação por bases na produtividade da borracha foram avaliados por regressão polinomial. O programa estatístico utilizado na análise dos dados foi o SAS (SAS Institute, 1996).

\section{Resultados e Discussão}

A análise conjunta dos valores de $\mathrm{pH}$, das concentrações de $\mathrm{Ca}, \mathrm{Mg}, \mathrm{H}+\mathrm{Al}, \mathrm{SB}$ e do $\mathrm{V}$ não mostrou interação significativa da época de amostragem do solo e as doses de calcário em todas as profundidades avaliadas (Tabela 1), revelando que as variações nas propriedades químicas do solo foram semelhantes da primeira para a segunda época de amostragem. Por sua vez, a maior parte do calcário reagiu até os seis meses, correspondente à primeira coleta de solo.

Houve efeito significativo das doses de calcário, em relação ao $\mathrm{pH}, \mathrm{Ca}, \mathrm{Mg}$, bem como redução no $\mathrm{H}+\mathrm{Al}$, refletindo aumento da soma de bases e saturação por bases nas profundidades de $0,00-0,10$ e $0,10-0,20 \mathrm{~m}$, entretanto não houve alteração significativa na camada subsuperficial $(0,20-0,40 \mathrm{~m})$ do solo (Tabela 1 e Figura 1). Este fato provavelmente deve-se ao tempo de reação do calcário (16 meses) que não foi suficiente para atingir a sua reação máxima e promover os maiores benefícios, na neutralização da acidez, nas camadas subsuperfíciais do solo. Caires et al. (2000) aplicaram calcário em superfície em sistema plantio direto e observaram, na camada de $0,10-0,20 \mathrm{~m}$, reação máxima do corretivo aos 28-30 meses após a aplicação.

Houve movimento do calcário até a camada de 0,10-0,20 m, após 16 meses da sua aplicação, concordando com os estudos de Oliveira \& Pavan (1996), que evidenciaram movimento significativo na profundidade $0,10-0,20 \mathrm{~m}$. Há trabalhos sobre a baixa mobilidade do calcário no perfil do solo (Koch \& Estes, 1986), atingindo até $0,05 \mathrm{~m}$ de profundidade, cinco meses após aplicação superficial (Pires et al., 2003) e até 0,10 m, 12 meses após sua aplicação na superfície do solo (Caires et al., 1998). O movimento de partículas de calcário no perfil do solo, é explicado por diversos fatores, entre os quais destaca-se o de natureza física, como os canais deixados pela decomposição de raízes, em razão da atividade da micro e da macrofauna (Pearson et al., 1962). Outra forma de movimentação do calcário é de natureza química, pela formação de pares entre bases $(\mathrm{Ca}$ e $\mathrm{Mg}$ ) e dos ácidos orgânicos de alta solubilidade que propiciam o carreamento destes nutrientes às camadas subsuperfíciais do perfil (Santos, 1999). O movimento do Ca observado pode ser decorrente da manta orgânica sobre o solo do seringal que por meio de sua decomposição propiciaria a formação de ligantes orgânicos que formariam os complexos $\mathrm{CaL}^{0}$ ou $\mathrm{CaL}^{-}$(Miyazawa et al., 2001). Além desses compostos, pode haver formação de outros como $\mathrm{Ca}\left(\mathrm{HCO}_{3}\right)_{2}$ e $\mathrm{Mg}\left(\mathrm{HCO}_{3}\right)_{2}$ (Oliveira \& Pavan, 1996).

A melhoria na saturação por bases na camada superficial do solo, após a aplicação do calcário (Figura 1), foi suficiente para proporcionar aumento e decréscimo significativos dos teores de $\mathrm{Ca}$ e $\mathrm{Zn}$ foliar, respectivamente, 14 meses após a aplicação (Figura 2). O incremento do teor de Ca foliar $\left(7,1-8,0 \mathrm{~g} \mathrm{~kg}^{-1}\right)$ foi suficiente para atingir o nível considerado adequado para seringueira $\left(7,6-8,2 \mathrm{~g} \mathrm{~kg}^{-1}\right)$, segundo Malavolta et al. (1997). Isto pode ser explicado pelo aumento da concentração de Ca no solo, uma vez que o calcário é fonte deste nutriente. Além disso, a ciclagem de nutrientes faz com que o $\mathrm{Ca}$ e o $\mathrm{N}$ retornem em maiores quantidades ao solo com a queda das folhas da seringueira (Murbach et al., 2003). O aumento das doses de calcário, elevou o $\mathrm{pH}$ do solo e, conseqüentemente, reduziu a so-

Tabela 1. Análise de variância dos atributos químicos do solo aos seis e 18 meses após a aplicação de calcário.

\begin{tabular}{|c|c|c|c|c|c|c|}
\hline Fonte de variação & $\mathrm{pH}$ & $\mathrm{Ca}$ & $\mathrm{Mg}$ & $\mathrm{H}+\mathrm{Al}$ & SB & $\mathrm{V} \%$ \\
\hline Época (E) & $5,68 * *$ & $100,83^{* *}$ & $11,41 * *$ & $64,53^{*}$ & $259,90 * *$ & $585,21 * *$ \\
\hline Dose (D) & $0,83 * *$ & $86,20 * *$ & $26,65 * *$ & $307,77 * *$ & $223,56 * *$ & $995,81 * *$ \\
\hline Profundidade $(\mathrm{P})$ & $0,23 * *$ & $797,31 * *$ & $82,76^{* *}$ & $167,61 * *$ & $1.436,89 * *$ & $1.173,03 * *$ \\
\hline$E \times D$ & $0,07^{\mathrm{ns}}$ & $20,83^{\mathrm{ns}}$ & $5,43^{\mathrm{ns}}$ & $16,13^{\mathrm{ns}}$ & $64,46^{\mathrm{ns}}$ & $95,85^{\mathrm{ns}}$ \\
\hline$E \times P$ & $0,01^{\mathrm{ns}}$ & $6,01^{\mathrm{ns}}$ & $1,66^{\mathrm{ns}}$ & $2,61^{\mathrm{ns}}$ & $1,92^{\mathrm{ns}}$ & $11,41^{\mathrm{ns}}$ \\
\hline $\mathrm{D} \times \mathrm{P}$ & $0,09 * *$ & $65,78 * *$ & $16,44 * *$ & $12,84^{\mathrm{ns}}$ & $122,71 * *$ & $182,73 * *$ \\
\hline$E \times D \times P$ & $0,02^{\mathrm{ns}}$ & $4,19^{\mathrm{ns}}$ & $1,43^{\mathrm{ns}}$ & $4,22^{\mathrm{ns}}$ & $5,01^{\mathrm{ns}}$ & $30,24^{\mathrm{ns}}$ \\
\hline $\mathrm{CV}(\%)$ & 3,51 & 17,99 & 21,42 & 18,16 & 17,10 & 11,79 \\
\hline
\end{tabular}

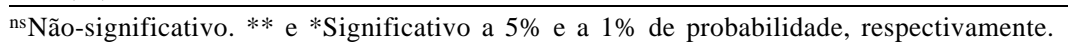


lubilidade do Zn do solo e na planta (Figura 1). Pereira \& Pereira (1987) também verificaram diminuição do Zn foliar com a calagem, em mudas de seringueira cultivadas em sacos de plásticos.

A calagem aumentou a produtividade da borracha seca da seringueira, no período avaliado (Figura 3), o que pode ser explicado pelos efeitos da calagem na correção da acidez e, conseqüentemente, aumento das bases, especialmente cálcio. A relação entre o teor foliar de $\mathrm{Ca}$ e a produtividade da borracha foi positiva (Produção $=-17.522+4.862,400 \mathrm{Ca}_{\text {foliar }}-310,900 \mathrm{Ca}^{2}$ foliar $)$. $\mathrm{O}$ teor de Ca foliar próximo a 7,8 $\mathrm{g} \mathrm{kg}^{-1}$ esteve associado à máxima produtividade de borracha seca. Além do efeito do Ca no crescimento radicular (Ritchey et al., 1982), esse nutriente é o segundo mais absorvido por essa espécie (Alves, 1987). Os resultados positivos da correção da acidez do solo na seringueira estão de acordo com as observações feitas por Bataglia et al. (1988) e
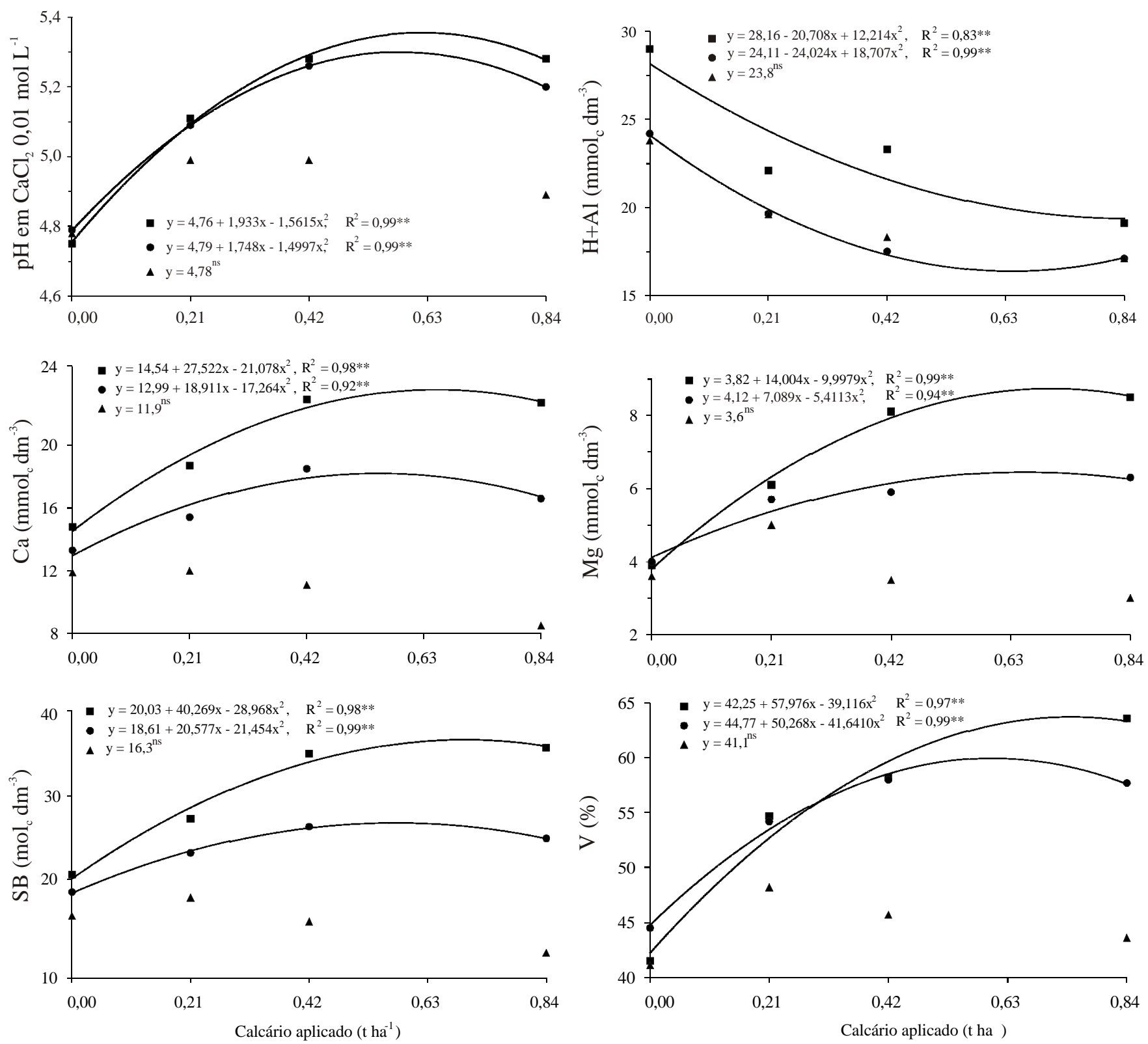

Figura 1. Efeito do calcário aplicado superficialmente nas características químicas, nas camadas de 0,00-0,10 m (ם), 0,10—0,20 m (•) e de 0,20-0,40 m ( $\mathbf{\Delta})$ de um Argissolo Vermelho-Amarelo cultivado com seringueira em produção. Dados médios de duas épocas de amostragem e cinco repetições. **Significativo a $1 \%$ de probabilidade pelo teste $\mathrm{F}$. 
Domingues (1994); entretanto, contrariam às de Reis et al. (1984).

A máxima produtividade $\left(1,55 \mathrm{t} \mathrm{ha}^{-1}\right)$ esteve associada à saturação por bases próxima a 57\% (Figura 4). Este valor está próximo ao sugerido por Cardoso (1992). Por sua vez, a saturação por bases acima deste valor (57\%) causou decréscimo da produtividade, possivelmente, por causa da menor disponibilidade de micronutrientes, a exemplo do $\mathrm{Zn}$ que teve seu nível foliar reduzido (Figura 2).

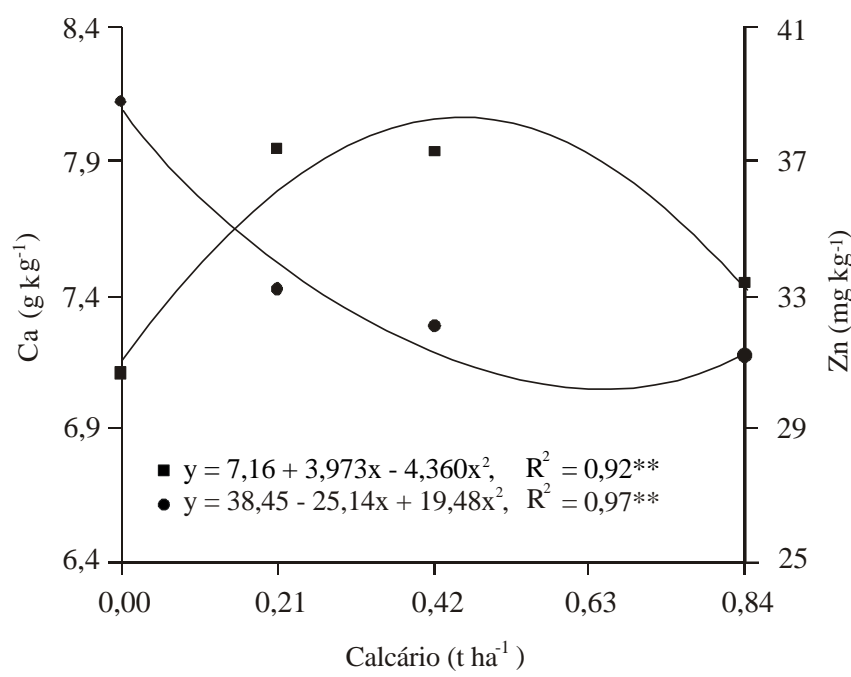

Figura 2. Efeito da aplicação superficial de calcário calcinado na concentração foliar de $\mathrm{Ca}(\boldsymbol{\square})$ e $\mathrm{Zn}(\bullet)$ aos 14 meses após a calagem de seringueiras adultas. **Significativo a $1 \%$ de probabilidade pelo teste $\mathrm{F}$.

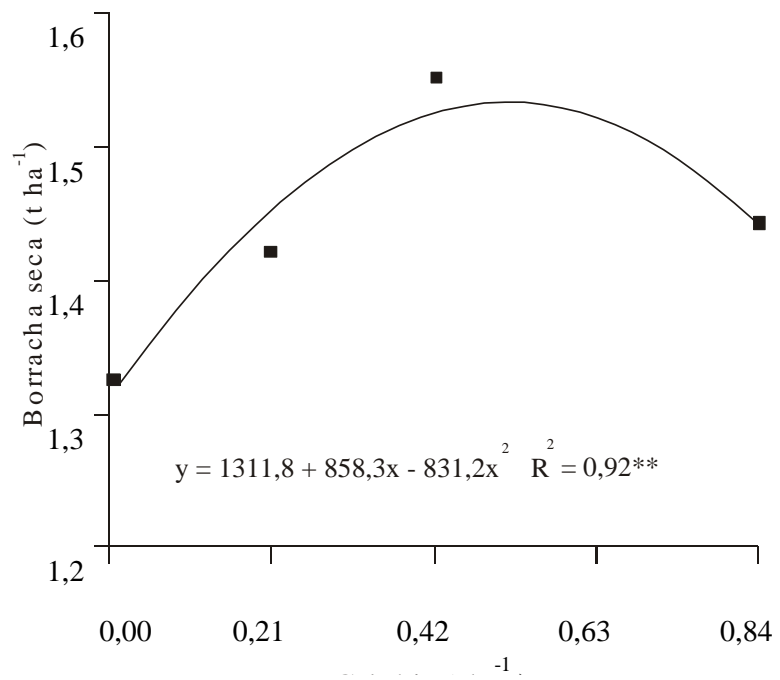

Figura 3. Efeito da aplicação superficial de calcário calcinado na produtividade de borracha seca de seringueiras adultas. Médias de dois anos de produtividade e cinco repetições. **Significativo a $1 \%$ de probabilidade pelo teste $\mathrm{F}$.

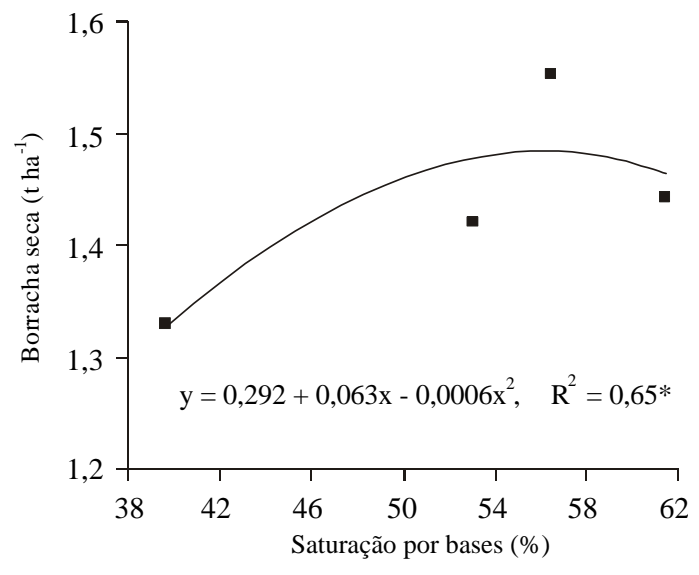

Figura 4. Relação entre a saturação por bases do solo (camada de $0,00-0,10 \mathrm{~m}$ ) e a produtividade de borracha seca da seringueira, cultivada em Argissolo Vermelho-Amarelo, em função da aplicação superficial de calcário calcinado. Média de dois anos. *Significativo a $5 \%$ de probabilidade pelo teste $\mathrm{F}$.

Pode-se considerar a máxima produtividade $\left(1,55\right.$ t ha $\left.^{-1}\right)$ obtida como alta, pois Bataglia et al. (1988) classificaram como altos os valores de produtividade acima de 1,5 t ha-1. Produtividades de 1,23 e 1,42 $\mathrm{t} \mathrm{ha}^{-1} \mathrm{de}$ borracha seca foram obtidas por Gonçalves et al. (2000, 2002), respectivamente, para o clone RRIM 600.

\section{Conclusões}

1. A aplicação de calcário na superfície do solo em seringal em produção neutraliza a acidez do solo e aumenta a saturação por bases até a camada de $0,20 \mathrm{~m}$, melhorando a nutrição da planta e a produtividade de borracha seca.

2. A produtividade máxima de borracha está associada à saturação por bases de $57 \%$ e teor foliar de Ca de $8,0 \mathrm{~g} \mathrm{~kg}^{-1}$.

\section{Referências}

ALVES, R.N.B. Níveis de nitrogênio, fósforo, potássio e magnésio para produção de porta-enxertos de seringueira (Hevea spp.) no Amapá. 1987. 79p. Dissertação (Mestrado) - Universidade Federal de Lavras, Lavras.

BATAGLIA, O.C.; CARDOSO, M.; CARRETERO, M.V. Situação nutricional de seringais produtivos no Estado de São Paulo. Bragantia, v.47, p.109-123, 1988.

BATAGLIA, O.C.; DECHEN, A.R.; SANTOS, W.R. Diagnose visual e análise de plantas. In: DECHEN, A.R.; BOARETTO, A.E.; VERDADE, F.C. (Ed.). Adubação, produtividade e ecologia. Campinas: Fundação Cargill, 1992. p.369-393. 
BATAGLIA, O.C.; FURLANI, A.M.C.; TEIXEIRA, J.P.F.; FURLANI, P.R.; GALLO, J.R. Métodos de análise química de plantas. Campinas: Instituto Agronômico, 1983. 48p. (Boletim Técnico, 78).

BUENO, N. Quantidade de alumínio no substrato afetando o desenvolvimento, a sintomatogia de toxicidade, a concentração e o acúmulo de macro e micronutrientes em seringueira (Hevea spp.). 1987. 92p. Tese (Doutorado) - Escola Superior de Agricultura Luiz de Queiroz, Piracicaba.

CAIRES, E.F.; BANZATTO, D.A.; FONSECA, A.F. Calagem na superfície em sistema plantio direto. Revista Brasileira de Ciência do Solo, v.24, p.161-169, 2000.

CAIRES, F.E.; CHVEIRI, A.W.; MADRUGA, E.F.; FIGUEIREDO, A. Alterações de características químicas do solo e resposta da soja ao calcário e gesso aplicado na superfície em sistema de cultivo sem preparo do solo. Revista Brasileira de Ciência do Solo, v.22, p.27-34, 1998.

CARDOSO, M. Seringueira. In: RAIJ, B. van; SILVA, N.M.; BATAGLIA, O.C.; QUAGGIO, J.A.; HIROCE, R.; CANTARELLA, H.; BELINAZZI JÚNIOR, R.; DECHEN, A.R.; TRANI, P.E. (Ed.). Recomendação de adubação e calagem para o Estado de São Paulo. Campinas: Instituto Agronômico, 1992. 285p. (Boletim Técnico, 100).

DOMINGUES, F.A. Nutrição mineral e crescimento de seringais em início de explotação no Estado de São Paulo. 1994. 59p. Dissertação (Mestrado) - Escola Superior de Agricultura Luiz de Queiroz, Piracicaba.

GONÇALVES, P.S.; MARTINS, A.L.M.; FURTADO, E.L.; SAMBUGARO, R.; OTTATI, E.L.; ORTOLANI, A.A.; GODOY JÚNIOR, G. Desempenho de clones de seringueira da série IAC 300 na região do planalto de São Paulo. Pesquisa Agropecuária Brasileira. v.37, p.131-138, 2002.

GONÇALVES, P.S.; SOUZA, S.R.; BRIOSCHI, A.P.; VIRGENS FILHO, A.C.; MAY, A.; ALARCON, R.S.C. Efeito da freqüência de sangria e estimulação no desempenho produtivo e econômico de clones de seringueira. Pesquisa Agropecuária Brasileira, v.35, p.1081-1091, 2000.

KOCH, D.W.; ESTES, G.O. Liming rate and method in relation to forage establishment: crop and soil chemical responses. Agronomy Journal, v.78, p.567-571, 1986

MALAVOLTA, E.; VITTI, G.C.; OLIVEIRA, S.A. Avaliação do estado nutricional das plantas: princípios e aplicações. 2.ed. Piracicaba: Potafos, 1997. 319p.

MIYAZAWA, M.; FRANCHINI, J.C.; VILA, F.J.G.; PAVAN, M. Rapid transformations of plant water soluble organic compounds in relation to cation mobilization in an acid Oxisol. Plant and Soil, v.231, p.55-63, 2001.

MURBACH, M.R.; BOARETTO, A.E.; MURAOKA, T. Ciclagem de nutrientes em um seringal do clone RRIM 600. Scientia Agricola, v.60, p.353-357, 2003.

MURBACH, M.R.; BOARETTO, A.E.; MURAOKA, T.; SILVEIRA, R.I.; BOARETTO, R.M. Adubação NPK e produção de borracha seca pela seringueira (Hevea brasiliensis). Scientia Agricola, v.56, p.71-76, 1999.
OLIVEIRA, E.L.; PAVAN, M.A. Control of soil acidity in no-tillage system for soybean production. Soil and Tillage Research, v.38, p.47-57, 1996.

PEARSON, R.W.; ABRUNA, F.; VICE-CHANCES, J. Effect of lime and nitrogen applications on downward movements of calcium and magnesium in two humid soils of Puerto Rico. Soil Science, v.93, p.77-82, 1962.

PEREIRA, A.V.; PEREIRA, E.B.C. Respostas de porta-enxertos de seringueira à calagem. Revista Brasileira de Ciência do Solo, v.11, p.333-336, 1987.

PIRES, F.R.; SOUZA, C.M.; QUEIROZ, D.M.; MIRANDA, G.V.; GALVÃO, C.C. Alteração de atributos químicos do solo e estado nutricional e características agronômicas de plantas de milho, considerando as modalidades de calagem em plantio direto. Revista Brasileira de Ciência do Solo, v.27, p.121-131, 2003.

PRADO, R.M. Efeito da calagem no desenvolvimento, no estado nutricional e na produção de frutos da goiabeira e da caramboleira. 2003. 68p. Tese (Doutorado) - Faculdade de Ciências Agrárias e Veterinárias, Jaboticabal.

RAIJ, B. van; ANDRADE, J.C.; CANTARELLA, H.; QUAGGIO, J.A. Análise química para avaliação da fertilidade dos solos tropicais. Campinas: Instituto Agronômico, 2001. 285p.

RAIJ, B. van; CANTARELLA, H.; QUAGGIO, J.A.; FURLANI, A.M.C. (Ed.). Recomendações de adubação e calagem para o Estado de São Paulo. 2.ed. Campinas: Instituto Agronômico, 1996. 255p. (Boletim Técnico, 100).

REIS, E.L.; SANTANA, C.J.; CABALA-ROSAND, P. Influência da calagem e adubação na produção da seringueira no sul da Bahia. Revista Theobroma, v.14, p.33-44, 1984.

RITCHEY, K.D.; SILVA, J.E.; COSTA, U.F. Calcium deficiency in clayey B horizons of savanna oxisols. Soil Science, v.133, p.378$382,1982$.

SANTOS, J.C.F. Mobilização de cálcio e alumínio em solos ácidos por compostos orgânicos hidrossolúveis de resíduos vegetais. 1999. 72p. Tese (Doutorado) - Escola Superior de Agricultura Luiz de Queiroz, Piracicaba.

SAS INSTITUTE (Cary, Estados Unidos). SAS/STAT procedure guide for personal computers. $5^{\text {th }}$ ed. Cary, 1996. 1686p.

SCHWENGBER, D.R. Efeitos do gesso e calcário na relação raiz/parte aérea e teores foliares de nutrientes em tocos de seringueira (Hevea spp. clone IAN-873). 1993. 126p. Dissertação (Mestrado) - Universidade Federal Lavras, Lavras.

SHORROCKS, V.M. Deficiências minerais em Hevea e plantas de cobertura associadas. Brasília: Sudhevea, 1979. 76p.

VIEGAS, I.J.M.; SAMPAIO, M.C.T.; COSTA CURTA, C.R.; THOMAZ, M.A.A.; PINHEIRO, E.; CARVALHO, J.G. Efeito das doses de cálcio na produção de matéria seca e na concentração de macronutrientes em plantas jovens de seringueira (Hevea spp.). Revista Ciências Agrárias, v.35, p.25-40, 2001.

VIRGENS FILHO, A.C.; MOREIRA, A.; CASTRO, P.R.C. Efeito da calagem e adubação da seringueira no estado nutricional e produção da borracha seca. Pesquisa Agropecuária Brasileira, v.36, p.10191026, 2001.

Recebido em 17 de outubro de 2003 e aprovado em 16 de fevereiro de 2004 